\title{
Percutaneous Biliary Drainage
}

\author{
Brian Funaki, M.D. ${ }^{1}$
}

\begin{abstract}
$\mathrm{P}_{\text {ercutaneous biliary drainage was one of the first }}$ nonvascular interventional radiology procedures to be widely used, and it continues to be performed today despite advances in endoscopic drainage. Currently, the procedure is usually reserved as a second-line therapy when endoscopic drainage is not feasible (e.g., due to prior biliary enteric anastomosis) or when endoscopic drainage fails.
\end{abstract}

\section{PROCEDURE}

\section{Preprocedure}

In all patients, any coagulopathy should be corrected prior to procedures when possible. All patients are prepped and draped in standard fashion. Preprocedural antibiotics are important: Piperacillin/tazobactam, $4.5 \mathrm{~g}$ $\mathrm{IV}$, may be given the morning of the procedure and continued for up to 2 days in appropriate individuals. Ciprofloxacin can be given in patients with an allergy to penicillins and cephalosporins. Additionally, intravenous fluid administration is performed before and after procedures because dehydration is common in this patient population.

\section{Procedure}

Moderate sedation can be performed with fentanyl and midazolam, although this will be insufficient in some patients. Deep sedation will be necessary in many patients, and in our hospital, this is performed by anesthesiologists. Some interventional radiologists favor a left-sided approach, but I typically begin on the right side. Imaging studies such as computed tomography are almost always available currently and are very helpful to plan the procedure. If perihepatic ascites is present, it should be drained before the procedure. (In children, we

\footnotetext{
${ }^{1}$ Section of Vascular and Interventional Radiology, University of Chicago Hospitals, Chicago, Illinois.

Address for correspondence and reprint requests: Brian Funaki, M.D., Section of Vascular and Interventional Radiology, University of Chicago Hospitals, 5840 S. Maryland Avenue, MC 2026, Chicago, IL 60637.
}

often use real-time ultrasound guidance for needle puncture, but in adults in a right-sided puncture, I simply perform a cursory scan at the intended puncture site to ensure no unexpected findings are present. Often, I use ultrasound for left-sided punctures in adults aiming for the segment 3 portal triad.) The intended puncture site is then marked using a hemostat and the patient is instructed to inspire deeply with fluoroscopic monitoring. The puncture site is chosen to be as cephalad as is allowable but caudal to the costophrenic angle to prevent pulmonary complications such as pneumothorax or empyema. I use an AccuStick introducer set (Boston Scientific, Natick, MA) with a 0.018-inch guidewire with a hydrophilic tip (V18 control wire; Boston Scientific) for access.

Local anesthetic is infiltrated deeply at the puncture site and a small skin nick is made with a scalpel. Punctures are directed slightly cephalad and the needle is advanced until the tip is a few centimeters to the right of the spine at the approximate T12 level. It is important to remember not to advance the needle too far in a cephalad direction because subsequent tube placement can be quite difficult if the path assumes the shape of a steep arch. The inner stylet is then withdrawn and the needle is slowly withdrawn while injecting $50 \%$ contrast until a duct is opacified (Fig. 1A,B). It is important to collimate the beam to the smallest reasonable area possible because these procedures can result in a large radiation dose for both patient and radiologist.

If the needle is withdrawn to nearly the capsule and a duct has not yet been opacified, the stylet is replaced and the needle is readvanced into the liver in a slightly different course. This is repeated for at least 10 passes or until a duct is entered. If after 10 or so passes, a duct has not been entered, a different puncture site on the skin may be chosen. If the patient has dilated intrahepatic ducts, in most procedures, a suitable duct for 
A
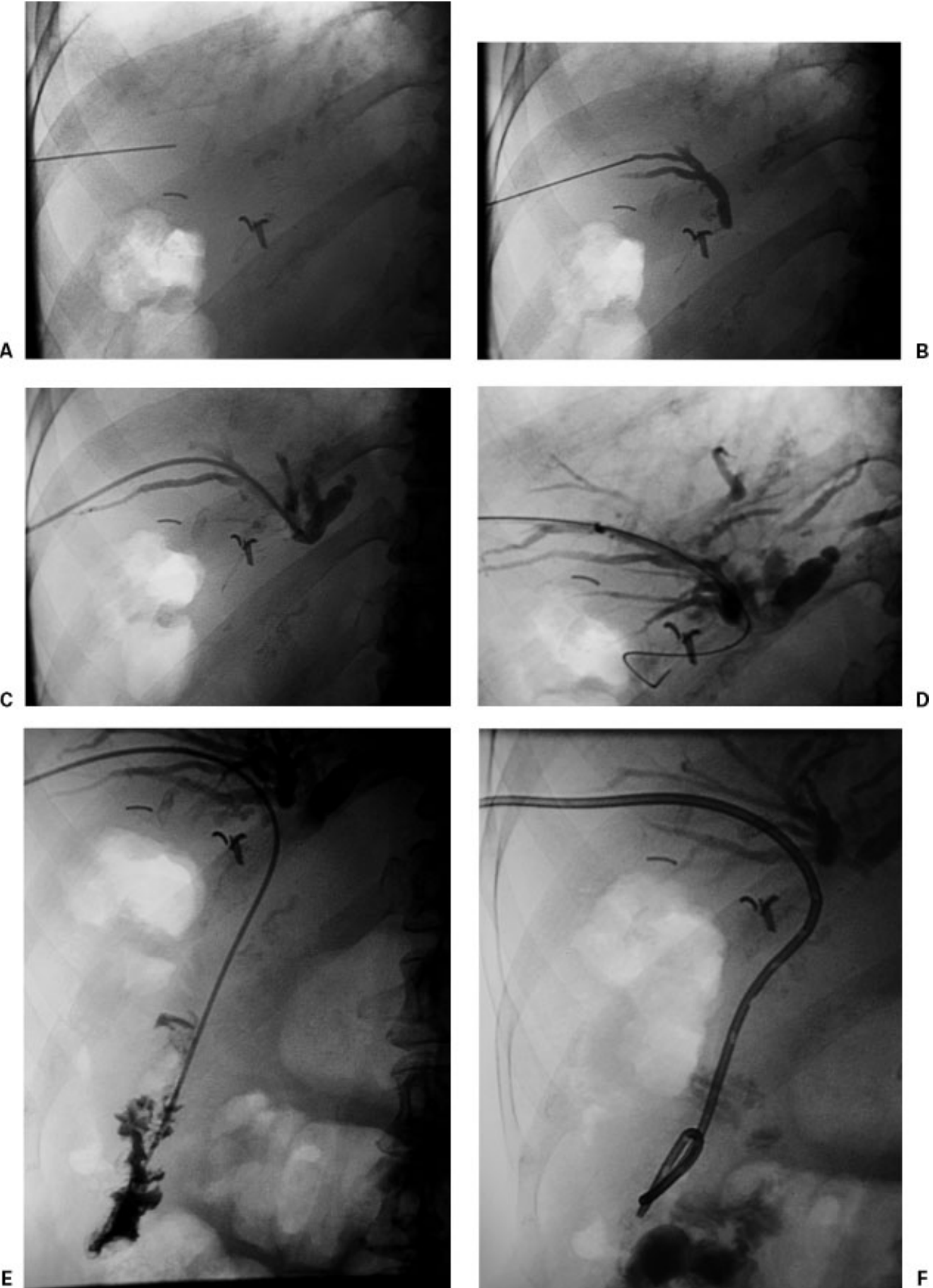

Figure 1 Percutaneous transhepatic biliary drainage in a 71-year-old woman with pancreatic carcinoma. (A) Fluoroscopic image shows needle being retracted in the liver after initial puncture. (B) Fluoroscopic image shows peripheral bile duct opacification. (C) Fluoroscopic image demonstrates 6F outer dilator of the AccuStick kit (Boston Scientific, Natick, MA) in the central bile ducts. (D) Fluoroscopic image shows 5F Kumpe (Cook, Bloomington, IN) catheter and guidewire advanced across the site of obstruction to expected region of small bowel. (E) Fluoroscopic image shows the 5F Kumpe catheter has been advanced into the small bowel. (F) Final fluoroscopic image shows $8 \mathrm{~F}$ biliary drainage catheter in good position.

access will be entered after a few passes through the liver. In patients with nondilated ducts, it may be difficult or impossible to access a duct. As a rule of thumb, if you have to ask yourself the question, "Am I in a bile duct?" then you are not in a bile duct.
Once a duct is opacified, a small amount of bile is aspirated and a similar amount of contrast is injected. It is important not to overdistend the ductal system in patients who are obstructed because this often results in sepsis. In rare cases, the opacified duct will be unsuitable 
(due to central location or angulation) for tube placement and a second puncture will need to be made using the first needle as a means to opacify suitable ducts. After a suitable duct is entered, an 0.018-inch hydrophilictipped rigid guidewire is advanced into the collecting system and if possible into common bile duct and small bowel. The needle is removed and coaxial 4 and $6 \mathrm{~F}$ dilators are inserted over the 0.018 -inch guidewire (Fig. 1C). The inner diameter of the inner $4 \mathrm{~F}$ dilator is designed to accept an 0.018-inch guidewire. When necessary, the dilators are exchanged for a short $5 \mathrm{~F}$ catheter (e.g., Kumpe; Cook, Bloomington, IN) and hydrophilic guidewire (Fig. 1D), which are manipulated into the small bowel (Fig. 1E). If this proves difficult, attempts are aborted after 5 to 10 minutes and the patient is brought back to the interventional radiology suite at a later date for further attempts.

A rigid 0.035-inch guidewire (Amplatz superstiff guidewire; Boston Scientific, Natick, MA) is inserted through the catheter and the tract is serially dilated using 7 and 9F dilators. An 8F biliary drainage catheter (Boston Scientific) with an inner metal stiffener is then advanced over the guidewire (if the small bowel has been accessed) and positioned with side holes bridging the obstruction but not too far peripheral in the liver (Fig. 1F). If the obstruction was not traversed, a $8 \mathrm{~F}$ Dawson-Mueller catheter (Cook, Bloomington, IN) is positioned in the intrahepatic ducts. The tube is then left to bag drainage.

\section{Postprocedure}

Biliary output should be charted. In most patients, bile will be blood tinged initially but should clear overnight. In patients who are obstructed (the majority), I wait 1 to 2 days before performing diagnostic cholangiography if that is necessary. At that time, plastic or metallic stents can be deployed as indicated. Most often, we place metal stents for malignant obstruction.

\section{DISCUSSION}

Occasionally, there is clear indication for either left or right biliary drainage (or both), but in many instances, the choice of a right or left access is personal preference. There are strong advocates of each approach and advantages and disadvantages to each. Advantages of left-sided tubes include ease of management for $\mathrm{pa}^{-}$ tients, improved patient comfort, and the ability to use ultrasound for guidance during the procedure. Pulmonary complications, which are rare with right-sided approaches, are essentially absent when a left-sided approach is used. These advantages are offset by increased hand radiation to the operator (particularly if ultrasound guidance proves difficult) and an inability to access the left lobe easily in some patients due to a "high" location under the xiphoid. Moreover, interposed bowel can prevent facile access to the left lobe in others. I find percutaneous drainage to be technically easier from the right lobe, more of the liver is drained from a right-sided approach, and it is therefore typically my preference to start on the right (Fig. 2).

\section{Nondilated Ductal Drainage}

Drainage in patients with nondilated ducts may be indicated in patients with bile duct injuries (e.g., laparoscopic cholecystectomy injury) or in liver transplant patients in whom ducts may not dilate even in the presence of obstruction. In our published experience, we have been able successfully to place a biliary drainage catheter in $\sim 90 \%$ of patients with nondilated ducts. In our hospital, most of these patients are liver transplant recipients. We are typically quite aggressive in our attempts, and as many as 50 to 75 passes are made into the liver. Bleeding is rarely a problem in these patients due to abundant scar tissue that surrounds the transplant. Cope has also described a technique for obtaining access to nondilated ducts with a 25- or 27-gauge needle used coaxially through a $21-$ or 22 -gauge needle.
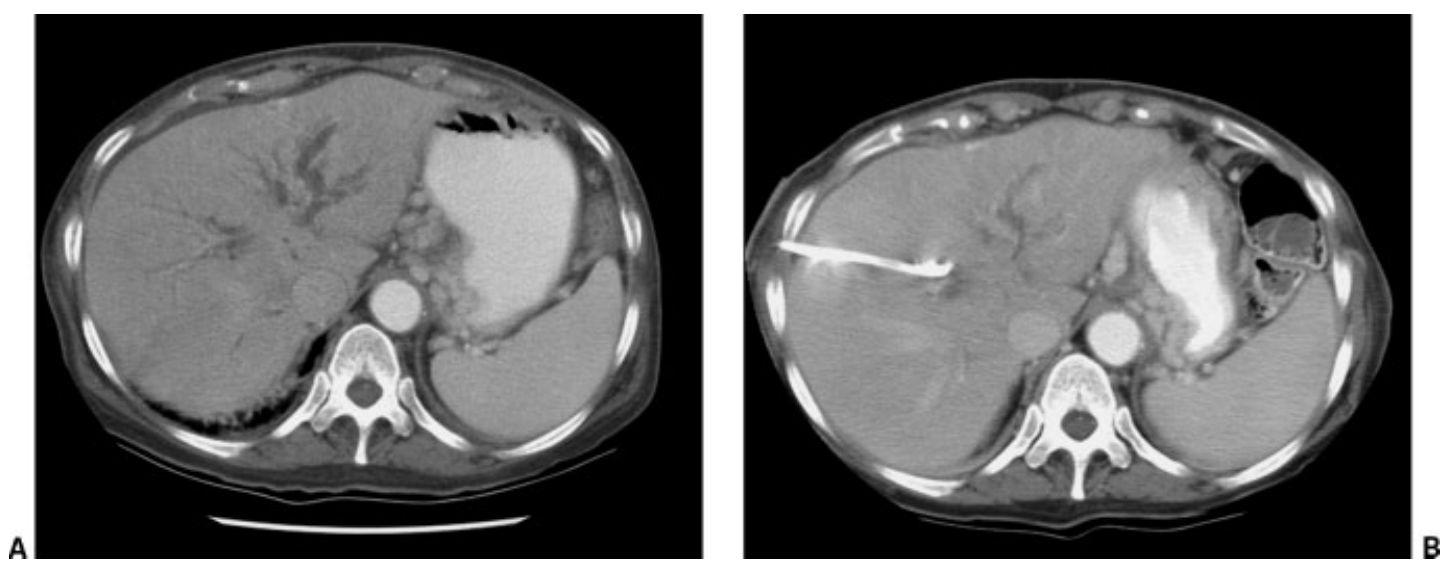

Figure 2 Computed tomography (CT) before (A) and after (B) percutaneous drainage. (A) CT image demonstrates intrahepatic biliary ductal dilatation and trace perihepatic ascites. (B) CT image after drainage shows decompression of biliary ducts. 


\section{Long-Term Tube Drainage}

In general, we attempt to provide definitive management within a week of tube insertion. In some patients, metallic stents are deployed; in others, tube drainage will be required for life. In those patients with long-term indwelling tubes, patients are instructed to flush the tube with 5 to $10 \mathrm{~mL}$ of water daily, and tubes are exchanged every 6 to 8 weeks. In some patients, exchange every 3 to 4 weeks will be necessary. If patients have fever, pain, or other tube dysfunction, they return for a cholangiogram and tube exchange.

\section{Stent Insertion for Malignant Obstruction}

I typically insert metal stents for palliation of malignant obstructions that are not amenable to surgical resection. Currently I use uncovered self-expanding stents such as the Wallstent (Boston Scientific, Natick, MA), although I have also used covered stents in some patients. The debate between covered and uncovered stents is unresolved in the literature. Some studies support and others refute the use of covered stents. Several studies have demonstrated improved long-term patency when covered stents are used with a slight increase in the incidence of cholecystitis and pancreatitis. Because covered stents are usually twice as costly as uncovered stents, I have refrained from using them regularly until more convincing data showing their superiority are available.

One common question relates to timing of tube removal after stent placement. In our hospital, internalexternal drainage is performed overnight after stent placement. The patients are then instructed to cap the tube for 2 days. If this is tolerated, they return for a cholangiogram, which is performed by removing the tube over a guidewire and inserting a vascular sheath with a sidearm that is used to perform cholangiography. If there is any question regarding the adequacy of drainage, a Dawson-Mueller catheter (Cook, Bloomington, IN) is left in place capped above the stent, and the patient is sent home for 3 to 4 days for a trial of internal drainage. If this is well tolerated, the tube is removed. We do not perform biliary manometry.

\section{Complications}

A variety of complications may occur during and after percutaneous biliary drainage:
- Sepsis: Supportive therapy is initiated including IV fluids and oxygen. We often administer additional antibiotics (such as Unasyn) and meperidine, $50 \mathrm{mg} \mathrm{IM}$.

- Bright red pulsatile blood from tube: If bright red pulsatile blood is coming from the tube or puncture site, a hepatic angiogram is performed to evaluate for either a hepatic artery-to-portal vein fistula or hepatic artery pseudoaneurysm. If the injury is not immediately apparent, it is often helpful to repeat the angiogram after the tube has been removed over a guidewire. If an arterial injury is found, embolization is performed using Gelfoam and coils. Typically, a subselective embolization is preferred, but in most patients a more proximal embolization may suffice (hepatic arteries are end arteries) if necessary when distal catheterization proves difficult.

- Dark red nonpulsatile blood: This usually indicates a hepatic or portal vein has been traversed. The tube is removed over a guidewire and a vascular sheath is inserted. A "tract-o-gram" is performed by injecting the sidearm of the sheath. If a venous injury is found, the tube is upsized (e.g., from 8 to $10 \mathrm{~F}$ ) and positioned so that none of the side holes are in close proximity to the injury. This typically succeeds in tamponading the bleeding. In rare instances, a second puncture site is necessary and the initial tube track is embolized with Gelfoam pledgets after tube removal.

- Bile leaking around the tube with or without fever and/or pain: This typically indicates that the tube has been inadvertently retracted or kinked or obstructed distally. In any case, the tube must be exchanged over a guidewire for a new one.

\section{SUGGESTED READINGS}

Burke DR, Lewis CA, Cardella JF, et al; Society of Interventional Radiology Standards of Practice Committee. Quality improvement guidelines for percutaneous transhepatic cholangiography and biliary drainage. J Vasc Interv Radiol 2003;14:S243S246

Cope C. Usefulness of a percutaneous transhepatic coaxial micropuncture needle technique in patients with nondilated peripheral intrahepatic ducts. AJR Am J Roentgenol 2003;181: 1017-1020

Funaki B, Zaleski GX, Straus CA, et al. Percutaneous biliary drainage in patients with nondilated intrahepatic bile ducts. AJR Am J Roentgenol 1999;173:1541-1544 